\title{
RELACIÓN ENTRE ALEXITIMIA Y HOSTILIDAD: ¿UNA ASO- CIACIÓN INFLUIDA POR EL MALESTAR PSICOLÓGICO?
}

\author{
B. Rueda \\ A.M. Pérez-García \\ P. Sanjuán \\ M.A. Ruiz
}

Departamento de Psicología de la Personalidad, Evaluación y Tratamientos Psicológicos. Facultad de Psicologia-UNED

\section{RESUMEN}

Este artículo examinó las relaciones entre la alexitimia y sus dimensiones (dificultad para identificar sentimientos, dificultad para describirlos, y pensamiento orientado al exterior) con el estrés psicológico (vulnerabilidad y estado depresivo) y la hostilidad (ira manifiesta, ira suprimida, cinismo y rumiación) Además estudió si la asociación de la alexitimia con la hostilidad estaba influida por el estrés psicológico.

436 participantes completaron medidas de alexitimia, malestar psicológico y hostilidad.

La alexitimia correlacionó positivamente con el estrés psicológico, y predijo todos los criterios elegidos. La dificultad en identificar y describir sentimientos predijeron la ira manifiesta y suprimida, y el cinismo. La rumiación fue explicada sólo por la dificultad en describir sentimientos. El estrés psicológico influyó

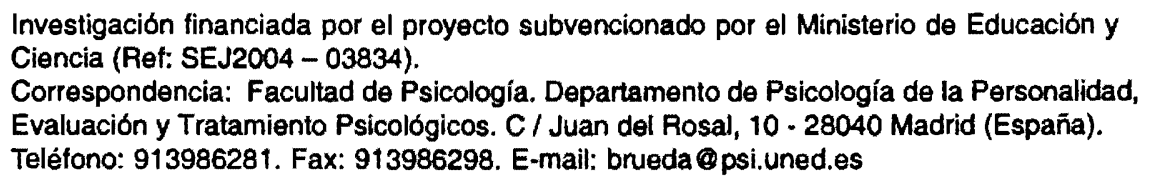


sobre la contribución de la alexitimia al enfado manifiesto y la rumiación. Este efecto fue menor para la ira suprimida y el cinismo. Se discutió el déficit en el manejo del enfado y la tendencia al estrés en individuos alexitímicos.

Palabras clave: ALEXITIMIA, HOSTILIDAD, VULNERABILIDAD Y DEPRESIÓN.

\section{SUMMARY}

This article analyzes the relationships between alexithymia and its dimensions (difficulty identifying feelings, difficulty describing them, and external orientated thinking) with psychological distress (vulnerability and depressive state) and hostility (anger-in, anger-out, cynicism and rumination). It also studied whether the association of alexithymia with hostility was influenced by psychological distress. Four hundred and thirtysix participants completed measures of alexithymia, psychological distress and hostility. Alexithymia positively correlated with psychological distress, and it predicted all the selected outcomes. Difficulty to identify and to describe feelings predicted anger-out, anger-in and cynicism. Rumination was only explained by difficulty in describing feelings. Psychological distress influenced the contribution of alexithymia to anger-out and rumination. This effect was lesser for anger-in and cynicism. The deficit in anger management and the proneness to stress in alexithimic individuals were discussed.

Key words: ALEXITHYMIA, HOSTILITY, VULNERABILITY AND DEPRESSION.

\section{INTRODUCCIÓN}

A lo largo de las tres últimas décadas el constructo de alexitimia (Sifneos, 1972) ha sido objeto de un extensa investigación por sus implicaciones en el ámbito de la salud física y psicológica. La alexitimia hace referencia a una disposición cognitivo-afectiva que se caracteriza 
por tres dimensiones básicas: la dificultad para identificar los sentimientos y diferenciarlos de las sensaciones corporales que acompañan al arousal emocional; la pobreza para describir los sentimientos a los demás; y el empleo de un estilo cognitivo extremadamente operativo y dirigido al exterior (Bagby, Parker, y Taylor, 1994). Otros rasgos que también se han asociado con la alexitimia destacan la escasa capacidad para elaborar fantasías y deseos, los estallidos repentinos de llanto o cólera, la tendencia a actuar como forma de expresión emocional, y el mantenimiento de unas relaciones interpersonales distantes o dependientes (Bagby, Taylor y Parker, 1997; Lesser, 1981).

Diferentes estudios han puesto de manifiesto la relación de la alexitimia con problemas de adicción (Helmers y Mente, 1999), estrés postraumático (Krystal, 1982) y con una variedad de trastornos psicosomáticos (Fortune, Richards, Giffiths y Main, 2002; Posse, Backenroth-Ohsako, Häkanson y Hällström, 2001; Yücel, Kora, Özyalcín et al., 2001).

En la actualidad algunas líneas de investigación han comenzado a llamar la atención acerca de la posible influencia de la alexitimia sobre otros factores psicológicos, como la hostilidad (Ciarrochi, Scott, Deane y Heaven, 2003; Linden, Lenz y Stossel, 1996; Rief, Heuser y Fichter, 1996).

La hostilidad es un constructo multidimensional que abarca componentes afectivos, cognitivos y conductuales (Smith, Glazer, Ruiz y Gallo, 2004). El componente afectivo engloba un conjunto de emociones relacionadas con la ira o la irritabilidad. La ira, a su vez, puede expresarse de forma explícita (ira manifiesta), o puede contenerse (ira suprimida) (Spielberger, Johnson, Russell et al., 1984). El componente conductual de la hostilidad hace referencia a conductas de agresión y falta de cooperación, mientras que el componente cognitivo, denominado cinismo, abarca un conjunto de creencias negativas sobre las personas y la mala intencionalidad de sus acciones (Houston y Vavak, 1991). Dentro de esta dimensión también se ha incluido la rumiación (Caprara, 1986), la cual supone un pensamiento reiterativo sobre el posible daño sufrido, acompañado de un sentimiento de venganza. Al hilo de esta diferenciación multicomponente de la hostilidad, Pelechano $(2000)$ ha propuesto 
recientemente el cuestionario de hostilidad y malevolencia (Hostymal), cuya estructura factorial se apoya en tres dimensiones: la dureza en el trato social, el rencor y recelo paranoide, y la desconfianza hacia la naturaleza humana.

Desde un punto de vista teórico se puede presuponer que la alexitimia, al constituir un déficit que afecta a la modulación y expresión del afecto negativo, comporte una mayor inhibición de la expresión emocional. Este proceso de inhibición, a su vez, puede promover la inmovilización en la expresión de la ira, con la consiguiente rumiación sobre las acciones suprimidas y la visión hostil del transgresor.

Esta suposición teórica viene corroborada por investigaciones empíricas en las que se observa que la alexitimia favorece una mayor supresión de la ira. Así por ejemplo, los resultados ofrecidos por Linden y colaboradores (1996) reflejaron que los estudiantes que puntuaban en el tercil más alto de la escala TAS de alexitimia, en comparación con los grupos medio y bajo, presentaban las puntuaciones más altas en ira interiorizada. En cuanto a la ira manifiesta, los resultados muestran mayor inconsistencia. Algunos trabajos (Rief et al., 1996) han puesto de relieve que las personas alexitímicas presentan niveles elevados de hostilidad, mientras que otros han demostrado que la alexitimia no parece vincularse con la expresión manifiesta de la ira (Sandín, Chorot, Santed, y Jiménez, 1996; Waldstein, Kauhanen, Neumann, y Katzel, 2002). Las dimensiones de la alexitimia que se han asociado en mayor medida con la supresión de la ira y la rumiación, son las relativas a la dificultad para identificar y describir los sentimientos (Ciarrochi et al., 2003; Waldstein et al., 2002). Sin embargo, la dimensión del pensamiento orientado al exterior se ha mostrado en este sentido más ambigua. Waldstein y colaboradores (2002) constataron que esta dimensión no guardaba relación con los procesos de ira y hostilidad. Otras investigaciones, o bien han evidenciado una asociación negativa entre estos factores (Bagby et al., 1994), o bien han obviado la inclusión de esta faceta de la alexitimia como variable de estudio (Cairrochi et al., 2003).

Considerando este conjunto de datos, uno de los objetivos que ha tenido el presente estudio ha sido examinar cómo se asociaba y en qué medida contribuia la alexitimia, y cada una de sus tres dimensiones, a la presencia de la hostilidad, considerando dentro de este 
constructo la vertiente emocional (ira manifiesta y suprimida) y la cognitiva (cinismo y rumiación). Asimismo, para comprender cómo funcionaba la alexitimia en relación con las manifestaciones de hostilidad, una cuestión que también nos ha interesado ha sido la de determinar el grado en que estas asociaciones eran directas, o se encontraban influidas por otros factores, como la vulnerabilidad psicológica y el estado de ánimo disfórico.

En este sentido se ha evidenciado que características definitorias de la vulnerabilidad psicológica (Sinclair y Wallston, 1999), como la necesidad de aprobación, la desorganización ante las situaciones de estrés o la dependencia en las relaciones interpersonales, constituyen también correlatos de la alexitimia (Bagby et al., 1994; Haviland y Reise, 1996; Waldstein et al., 2002). No obstante hay que advertir que, hasta la fecha, no se ha llevado a cabo ningún estudio sobre la relación de la alexitimia y de sus diferentes facetas con el constructo específico de la vulnerabilidad psicológica. En claro contraste se encuentra el amplio volumen de estudios sobre alexitimia y depresión. En ellos la alexitimia aparece asociada de forma moderada y significativa con el trastorno depresivo, tanto en muestras de pacientes psiquiátricos (Handelsman, Stein, Bernstein et al., 2000; Luminet, Bagby y Taylor, 2001; Rief et al., 1996) como en muestras con población normal (Modestin, Furrer y Malti, 2004; Posse et al., 2001; Sandin et al., 1996). De las tres dimensiones que componen la alexitimia, la dificultad para identificar y discriminar los sentimientos parece que es la que tiene un mayor peso en la predicción de la depresión (Posse et al., 2001; Saarijärvi, Salminen y Toikka, 2001; Sandín et al., 1996), si bien algunos datos apoyan la superioridad predictiva de la dificultad para describir sentimientos (Eizaguirre, Saenz de Cabezón, Alda et al., 2004; Marchesi, Brusamonti y Maggini, 2000). Muchas de estas investigaciones, sin embargo, adolecen de haberse centrado en el estudio de la influencia de la alexitimia sobre la depresión una vez que ésta ha sido diagnosticada ya como trastorno, prestando menos atención a los efectos sobre el estado anímico depresivo.

A tenor de estas cuestiones, se hace palpable la necesidad de estudiar, por un lado, las relaciones de la alexitimia y de sus distintas facetas con la vulnerabilidad psicológica y el estado de ánimo depresivo; y, por otro, examinar la capacidad que estos dos factores 
pueden tener para modular las asociaciones de la alexitimia con la expresión de la ira, el cinismo y la rumiación. En consecuencia, los objetivos planteados en nuestra investigación fueron: (a) estudiar, en una muestra de adultos sanos, la relación de la alexitimia y de sus facetas (dificultad para identificar los sentimientos, dificultad para describirlos, y pensamiento orientado al exterior) con la vulnerabilidad psicológica y el estado de ánimo depresivo; (b) estudiar la contribución de la alexitimia y de sus componentes con respecto a la hostilidad, abarcando sus aspectos emocionales (ira suprimida e ira manifiesta) y cognitivos (cinismo y rumiación); y (c) determinar en qué medida la vulnerabilidad psicológica y el estado de ánimo depresivo podian afectar la relación de la alexitimia con la hostilidad.

Siguiendo estos objetivos establecimos, como primera hipótesis, que la alexitimia se asociaría de forma positiva con la vulnerabilidad psicológica y el estado de ánimo depresivo. Con respecto a las dimensiones de la alexitimia, establecimos que la dificultad para identificar los sentimientos sería la que mostrara mayor asociación con el estado de ánimo depresivo.

La segunda hipótesis sostuvo que la alexitimia tendría un peso positivo en la predicción de la ira suprimida, el cinismo y la rumiación, siendo las dimensiones de dificultad en identificar los sentimientos y dificultad en describirlos las que se vincularian en mayor medida con las variables anteriormente señaladas. En cuanto a la ira manifiesta, presumimos que tendría una modesta asociación con la alexitimia.

Por último planteamos como pregunta de investigación en qué medida la relación de la alexitimia con la hostilidad era directa, o estaba modulada en parte por la vulnerabilidad psicológica y el estado depresivo.

\section{MÉTODO}

\section{Participantes}

La muestra se seleccionó a partir de una población de adultos sanos que estaban inscritos en diferentes cursos universitarios. Se entregaron un total de 876 cuestionarios que contenian las medidas objeto de estudio. Una vez rellenado el cuestionario, los participantes debian devolverlo por correo a la facultad de psicologia. 
La muestra final estuvo compuesta por 436 participantes (49\%), de los cuales 327 eran mujeres y 101 hombres, habiendo 20 personas que no indicaron el género. La edad media fue de 36 años. El rango de edad estuvo comprendido entre los 17 y los 63 años.

Con respecto al estatus laboral, se obtuvo información de 416 participantes. Entre ellos un $55 \%$ ocupaba puestos de nivel intermedio o superior; un $13 \%$ se dedicaba a profesiones liberales; un $20 \%$ era estudiante; un $9 \%$ se ocupaba de las tareas del hogar; y un $3 \%$ estaba desempleado.

\section{Medidas}

Escala de Alexitimia de Toronto (Toronto Alexithymia Scale (TAS20); Bagby et al., 1994): La TAS-20 es una medida de alexitimia, creada a partir de la escala original TAS (Taylor, Ryan y Bagby, 1985). La TAS20 se compone de 20 ítems, valorados de 1 (nada) a 5 (mucho). Consta de tres subescalas referidas a los siguientes dominios: dificultad para identificar y distinguir los sentimientos de las sensaciones corporales asociadas con la activación emocional (7 ítems); dificultad para describir los sentimientos ( 5 ítems); y pensamiento dirigido al exterior (8 ítems). En nuestro estudio consideramos, tanto las puntuaciones en cada una de las tres subescalas, como la puntación global obtenida a partir de la suma de las subescalas.

La estructura trifactorial de la TAS-20 ha sido constatada por diferentes análisis confirmatorios (ver Taylor, Bagby y Luminet, 2000, para una revisión). Asimismo se ha informado sobre su validez interna y adecuada estabilidad en población clínica y normal (Bagby et al., 1994; Taylor, Bagby y Parker, 1997). Los coeficientes alphas obtenidos en esta investigación fueron elevados para la escala global (.84) y para las subescalas de dificultad en la identificación de sentimientos (.88) y dificultad en la descripción de sentimientos (.80). El coeficiente alpha fue substancialmente más bajo para la subescala de pensamiento orientado al exterior (.48).

Escala de Expresión de la Ira (Spielberger, Johnson, Russell et al., 1984): Este autoinforme se compone de 25 ítems y evalúa la expresión habitual del enfado. La escala se compone de tres dimensiones: la ira suprimida ( 7 ítems), que indica la tendencia a suprimir esta emoción 
cuando la persona se siente agredida por un estímulo externo o interno; la ira manifiesta (10 ítems), que alude a su expresión abierta ante las demás personas o contra los objetos; y el control de la ira ( 8 ítems), que se refiere a la expresión controlada de esta emoción. En este estudio sólo incluimos las subescalas de enfado suprimido y enfado manifiesto, en la medida en que estos dos estilos han sido objeto de estudio en el ámbito de la alexitimia (Sandín et al., 1996; Waldstein et al., 2002). Los ítems fueron evaluados a través de una escala de cuatro puntos, donde 1 = Casi nunca y $4=$ Casi siempre. La escala de expresión del enfado ha mostrado tener una adecuada consistencia interna (Knight, Chisholm, Paulin y Waal-Manning, 1988). En nuestra investigación, el coeficiente alpha para el enfado manifiesto fue notablemente elevado $(a=.78)$, siendo más bajo el obtenido para el enfado suprimido $(a=.58)$.

Escala de Hostilidad: Subescala de Cinismo (Cook y Medley, 1954; adaptación española de Bermúdez, Fernández y Sánchez-Elvira, 1992): Para medir la hostilidad cínica se empleó una versión reducida de la escala de Cook y Medley (1954), compuesta de 8 ítems. La hostilidad cínica se define como el conjunto de creencias y atribuciones que mantiene la persona sobre el comportamiento malintencionado y egoísta de los demás.

El formato de respuesta fue tipo Likert, oscilando la puntuación desde 0 (Totalmente falso) hasta 5 (Totalmente verdadero), de manera que una alta puntuación en la escala indicaba un grado elevado de cinismo. El coeficiente alpha de la escala fue apropiado (á =.84).

La escala de Disipación - Rumiación (DRS; Caprara, 1986): La DRS se compone de 20 ítems, de los cuales 5 son de control. La disipación y la rumiación son los polos opuestos de una dimensión, en la que la rumiación reflejaría el deseo de vengarse por una ofensa, y la disipación la tendencia a olvidarla. En el presente trabajo se utilizó una versión adaptada de la escala (Bermúdez, 1993), que no contenía los cinco ítems de control. El formato de respuesta fue tipo Likert (donde $0=$ Totalmente falso y $5=$ Totalmente verdadero). La consistencia interna de la escala fue bastante alta $(a ́=.91)$. Una elevada puntuación en el cuestionario señala un alto grado de rumiación.

Escala de Vulnerabilidad Psicológica (PVS; Sinclair y Wallston, 1999): Este instrumento mide el grado de vulnerabilidad psicológica, 
definida como la necesidad de dependencia y aprobación, el mantenimiento de un patrón de pensamiento perfeccionista y la realización de atribuciones negativas. La escala se compone de 6 ítems con un formato de respuesta tipo Likert con cinco puntos (desde $1=$ Nada hasta $5=$ Mucho). Una puntuación alta en la escala indica un nivel elevado de vulnerabilidad.

La PVS ha mostrado una consistencia interna alta en diferentes muestras, oscilando entre .71 y .86, y una adecuada estabilidad temporal (Sinclair y Wallston, 1999; Pérez-García, Rueda, Sanjuán y Ruiz, 2006). En nuestro estudio el coeficiente alpha obtenido fue .77.

Escala de Ansiedad y Depresión: subescala de depresión (HADS; Zigmond y Snaith, 1983): La HADS es una medida autoinformada que permite evaluar el estado de ánimo ansioso y/o deprimido en población no psiquiátrica. Cada una de las dos subescalas que componen la HADS constan de 7 ítems, oscilando el formato de respuesta entre 1 y 4 puntos. Este instrumento permite obtener una puntuación global en malestar emocional, y en las dimensiones de ansiedad y depresión. Puesto que los objetivos planteados en este estudio giraron en torno al papel del estado de ánimo depresivo, sólo tuvimos en cuenta la puntuación obtenida en la subescala de depresión.

Investigaciones previas han documentado la consistencia interna y alta estabilidad de la HADS (Martin y Thompson, 2000; Quintana, Padierna et al., 2003; Rueda, 2003). El coeficiente alpha de la subescala de depresión fue .81 en nuestra investigación.

\section{Análisis de datos}

En primer lugar se calcularon las correlaciones entre la alexitimia, y sus respectivas dimensiones, con la vulnerabilidad psicológica, el estado de ánimo depresivo, y con las variables criterio, es decir, con la ira manifiesta, la ira suprimida, el cinismo y la rumiación.

A continuación se llevaron a cabo una serie de análisis de regresión en bloque para examinar la contribución de la alexitimia y de sus facetas con respecto a las variables criterio.

Finalmente, para determinar si la relación de la alexitimia con las variables criterio estaba influida por la vulnerabilidad psicológica y el estado de ánimo depresivo, se realizaron una serie de análisis de 
regresión en bloque, siguiendo el procedimiento sugerido por algunos autores (Baron y Kenny, 1986). El nivel de significación mantenido en todos los análisis fue .05 .

\section{RESULTADOS}

\section{Análisis preliminares}

En la tabla 1 se muestran las medias, desviaciones típicas y rangos de puntuación obtenidos para cada una de las medidas.

Tabla 1. Estadisticos descriptivos y coeficientes alpha de las variables de estudio

\begin{tabular}{|l|c|c|c|c|c|}
\hline & M & DT & $\begin{array}{c}\text { Rango } \\
\text { Real }\end{array}$ & $\begin{array}{c}\text { Rango } \\
\text { Posible }\end{array}$ & $\alpha$ \\
\hline Alexitimia & & & & & \\
Global & 41,02 & 9,41 & $20-73$ & $20-100$ & .84 \\
Dificultad Identificar Sentimientos & 12,26 & 4,57 & $7-35$ & $7-35$ & .88 \\
Dificultad Describir Sentimientos & 11,26 & 3,91 & $5-22$ & $5-25$ & .80 \\
Pensamiento Orientado Exterior & 17,49 & 3,53 & $8-28$ & $8-40$ & .48 \\
\hline Hostilidad & & & & & \\
Ira Manifiesta & 17,91 & 4,38 & $10-35$ & $10-40$ & .78 \\
Ira Suprimida & 13,98 & 3,18 & $7-25$ & $7-28$ & .58 \\
Cinismo & 14,37 & 7,04 & $0-35$ & $0-40$ & .84 \\
Rumiación & 24,78 & 12,43 & $0-60$ & $0-75$ & .91 \\
\hline Vulnerabilidad Psicológica & 15,12 & 4,07 & $6-30$ & $6-30$ & .77 \\
\hline Estado Ánimo Depresivo & 10,61 & 3,56 & $7-26$ & $7-28$ & .81 \\
\hline
\end{tabular}

Para evaluar si existían diferencias de género en todas las variables consideradas, se llevaron a cabo una serie de ANOVAS univariados. Los resultados pusieron de manifiesto un estado de ánimo depresivo más alto en las mujeres ( $M=10.81)$, en comparación con los hombres $\left(M=9.91 ; F_{(1,427)}=4.96, p<.05\right)$; si bien estos puntuaron más alto en ira 
suprimida $\left(M_{\text {nomb }}=14.61, M_{\text {mul }}=13.71 ; F_{(1.427}=6.41, p<.05\right)$, cinismo $\left(M_{\text {nomb }}=15.61, M_{\text {mul. }}=13.88 ; F_{(1.427)}=4.68, p<.05\right)$ y rumiación $\left(M_{\text {nomb }}:=27.61, M_{\text {mul }}=23.78 ; F_{(1,427)}=7.46, p<.001\right)$.

Teniendo en cuenta estos datos, a la hora de realizar los análisis de regresión para predecir la ira suprimida, el cinismo y la rumiación, el género entró en las ecuaciones como una covariante.

Correlaciones entre la alexitimia, la vulnerabilidad psicológica y el estado de ánimo depresivo

Las intercorrelaciones entre todas las variables se presenta en la tabla 2. En primer lugar se aprecia una correlación positiva y moderada entre las subdimensiones de alexitimia, dato éste que vendría a reflejar la independencia conceptual entre las subescalas. No obstante, la asociación de las subescalas con la puntuación global de alexitimia fue alta, especialmente en el caso de la dificultad para identificar los sentimientos y la dificultad para describirlos.

En segundo lugar, como se habia especulado, la alexitimia correlacionó de forma positiva con la vulnerabilidad psicológica y el estado de ánimo depresivo, siendo la magnitud de los coeficientes de ambas correlaciones prácticamente semejante.

En cuanto a las subdimensiones de la alexitimia, la dificultad en identificar los sentimientos fue la faceta que se asoció en mayor medida con la vulnerabilidad psicológica y el estado de ánimo depresivo. La dificultad en describir los sentimientos y el pensamiento orientado al exterior también correlacionaron positivamente con estos dos factores, aunque el peso de las correlaciones fue menor.

\section{Relación de la alexitimia con las variables criterio}

Como se aprecia en la tabla 2, la alexitimia correlacionó positiva y significativamente con todas las variables criterio, aunque su conexión con la ira manifiesta fue notablemente menor.

Las tres facetas de la alexitimia también se asociaron positivamente con todas las variables criterio, a excepción del enfado manifiesto que sólo correlacionó positivamente con la dificultad para identificar los sentimientos. La relación de esta dimensión con la vulnerabilidad psicológica, así como la correlación entre la dificultad para describir los sentimientos y la ira suprimida, fueron las más elevadas. 


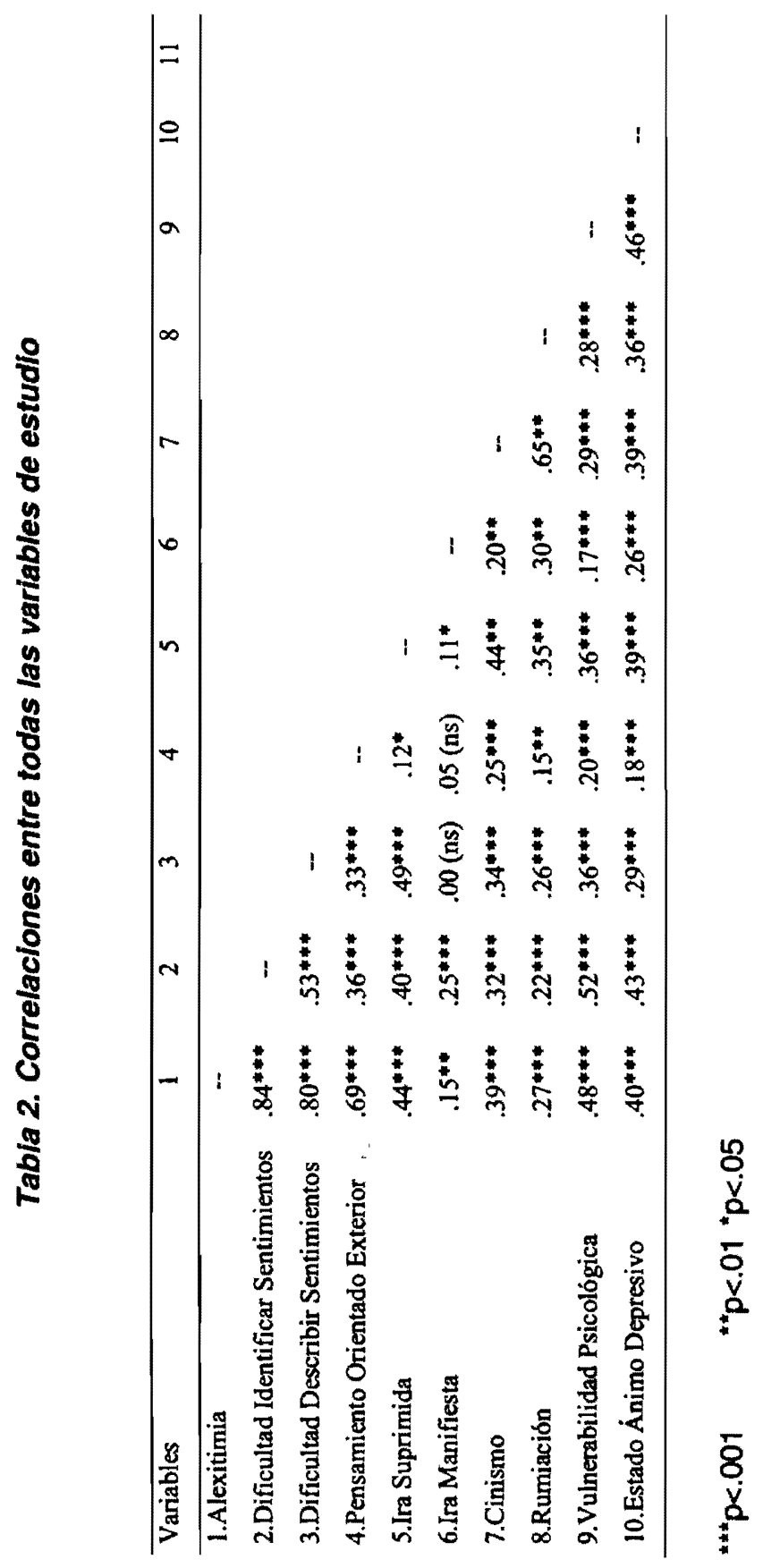


Por otra parte se aprecia que el pensamiento orientado al exterior es el componente de la alexitimia que menor relación tuvo con casi todas las variables criterio. Aún así las correlaciones establecidas fueron significativas, de lo cual puede inferirse que el mantenimiento de este tipo de patrón de pensamiento tiende a asociarse, en cierta medida, con la supresión del enfado y con un estilo rumiativo y hostil.

\section{Predicción de las variables criterio a partir de las dimensiones de la alexitimia}

A continuación se llevó a cabo una serie de análisis de regresión para determinar la capacidad predictiva de las dimensiones de alexitimia con respecto a la ira manifiesta, la ira suprimida, el cinismo y la rumiación. Para evitar el efecto de multicolinealidad, en esta primera serie de análisis no se incluyó la puntuación global de alexitimia.

Como reflejan los datos de la tabla 3 , la dificultad para identificar sentimientos y la dificultad para describirlos predijeron la mayor parte de los criterios considerados, corroborando así la hipótesis que habíamos planteado inicialmente.

En particular, la dificultad para describir los sentimientos emergió como el predictor más importante de la ira suprimida, el cinismo y la rumiación, siendo su peso positivo en todos los casos. Resulta llamativo por otra parte el hecho de que, en el caso de la ira manifiesta, esta faceta tuviera una contribución negativa.

La dificultad para identificar sentimientos también predijo positivamente la ira manifiesta, la ira suprimida y el cinismo. Con respecto a la rumiación su contribución fue sólo marginalmente significativa, aunque en la línea esperada.

En cuanto al pensamiento orientado al exterior, su capacidad predictiva fue menor que la mostrada por las otras dos facetas de la alexitimia, si bien explicó de forma significativa la ira suprimida (beta negativo) y el cinismo (beta positivo). En contraste, este criterio no resultó significativo en la predicción de la ira manifiesta y la rumiación.

En resumen, parece que, dentro de la constelación de los componentes de la alexitimia, la dificultad en la descripción de los sentimientos, seguida de la dificultad en identificarlos, son los factores que se asocian en mayor medida con la inhibición del enfado y al 
mantenimiento de creencias hostiles. El pensamiento orientado al exterior, sin embargo, parece relacionarse en menor medida, variando su contribución según el tipo de proceso cognitivo o afectivo que se trate.

Tabla 3.Análisis Múltiple de Regresión para predecir las diferentes manifestaciones de hostilidad a partir de las dimensiones de alexitimia

\begin{tabular}{|c|c|c|c|}
\hline Ira manifiesta & $\overline{\mathrm{R}^{2}}$ & gl & $\beta$ \\
\hline Dif. Identificar Sentimientos & .09 & $13,76^{* * *} 3,435$ & $.35^{* * *}$ \\
\hline Dif. Describir Sentimientos & & & $-.18 * *$ \\
\hline Pensam. Orientado exterior & & & $-.01(\mathrm{~ns})$ \\
\hline \multicolumn{4}{|l|}{ Ira suprimida } \\
\hline Género $^{\text {? }}$ & .28 & $40,51^{* * *} \quad 4,427$ & $-.10^{*}$ \\
\hline Dif. Identificar Sentimientos & & & $.23^{* * *}$ \\
\hline Dif. Describir Sentimientos & & & $.39^{* * *}$ \\
\hline Pensam. Orientado exterior & & & $-.11^{*}$ \\
\hline \multicolumn{4}{|l|}{ Cinismo } \\
\hline Género $^{Q}$ & .16 & $19,62 * * * \quad 4,427$ & $-.08+$ \\
\hline Dif. Identificar Sentimientos & & & $.18^{* *}$ \\
\hline Dif. Describir Sentimientos & & & $.20 * * *$ \\
\hline Pensam. Orientado exterior & & & $.10^{*}$ \\
\hline \multicolumn{4}{|l|}{ Rumiación } \\
\hline Género $^{2}$ & .09 & $10,51^{* * *} \quad 4,427$ & $-.12^{*}$ \\
\hline Dif. Identificar Sentimientos & & & $.11+$ \\
\hline Dif. Describir Sentimientos & & & $.18^{*}$ \\
\hline Pensam. Orientado exterior & & & .04 (ns) \\
\hline
\end{tabular}

\& 1 = Varón $2=$ Mujer $\quad * * * p<.001 \quad{ }^{* *} p<.01 \quad * p<.05 \quad+=.06$

Predicción de las variables criterio a partir de la alexitimia, la vulnerabilidad y el estado de ánimo depresivo 
La contribución de la alexitimia, la vulnerabilidad y el estado de ánimo depresivo en la predicción de las distintas variables criterio se detectó a través de ecuaciones de regresión en bloque (ver tablas 4 y 5$)$.

Los análisis de regresión pusieron de relieve, en primer lugar, que la relación de la alexitimia sobre la ira manifiesta y la rumiación estaba influida por la vulnerabilidad psicológica. En la primera ecuación de regresión, en la que la alexitimia había entrado como único predictor, se observó que esta variable predecía de forma positiva y significativa ambos criterios $(\beta=.15, p<.01$ para la ira manifiesta; $y \beta=.26, p<.001$ para la rurriación). Sin embargo, cuando se introdujeron la vulnerabilidad y el estado de ánimo depresivo junto con la alexitimia, el peso de ésta no fue significativo, siendo la vulnerabilidad psicológica la variable que explicó en mayor medida la variabilidad de la ira manifiesta $(\beta=.23, p<.001)$ y la rumiación $(\beta=.27, p<.001)$. El estado de ánimo depresivo, por su parte, sólo explicó parte de la varianza de la rumiación $(\beta=.15, p<.01)$.

En el caso de la ira suprimida y el cinismo, la alexitimia inicialmente predijo de forma positiva estos criterios $(\beta=.42, p<.001$ para la ira suprimida; y $\beta=.38, p<.001$ para el cinismo). A continuación, cuando se introdujeron conjuntamente la alexitimia, la vulnerabilidad y el estado de ánimo depresivo, los resultados indicaron que la alexitimia se mantenía como un predictor significativo, aunque su capacidad predictora había disminuido $(\beta=.26, p<.01$ para la ira suprimida; y $\beta=$ $.23, p<.001$ para el cinismo). La vulnerabilidad psicológica, por su parte, predijo también de forma positiva ambos criterios. Sin embargo, el estado de ánimo depresivo sólo contribuyó a explicar significativamente la variabilidad de la ira suprimida.

En resumen, a partir de estos análisis, se constató que el papel de la alexitimia era relevante en la predicción de la ira interiorizada y el cinismo. Sin embargo, la relación con la expresión abierta de la ira fue mayor en el caso de la vulnerabilidad psicológica que en el de la alexitimia. Con respecto al proceso de rumiación de pensamientos vengativos, se observó un efecto conjunto por parte de la alexitimia, la vulnerabilidad psicológica y el estado de ánimo depresivo. 
Tabla 4. Análisis mediacional en la predicción de la ira manifiesta y suprimida

\begin{tabular}{|l|cccc|}
\hline Ira manifiesta & $\mathrm{R}^{2}$ & $\mathrm{~F}$ & $\mathrm{gl}$ & $\beta$ \\
\hline Alexitimia & .02 & $9,46^{* *}$ & 1,435 & $.15^{* *}$ \\
\hline Vulnerabilidad Psicológica & .07 & $16.57^{* * *}$ & 2,435 & $.23^{* * *}$ \\
Estado Depresivo & & & & $.06(\mathrm{~ns})$ \\
\hline Alexitimia & .07 & $11.04^{* * *}$ & 3,435 & $.01(\mathrm{~ns})$ \\
Vulnerabilidad Psicológica & & & & $.23^{* * *}$ \\
Estado Depresivo & & & & $.05(\mathrm{~ns})$ \\
\hline Ira suprimida & & & & \\
\hline Género & & & & $-.10^{*}$ \\
Alexitimia & .19 & $51,25^{* * *}$ & 2,427 & $.42^{* * *}$ \\
\hline Género & & & & $-.17^{* * *}$ \\
Vulnerabilidad Psicológica & & & & $.28^{* * *}$ \\
Estado Depresivo & & & & $.25^{* * *}$ \\
\hline Género & .26 & $38,13^{* * *}$ & 4,427 & $-.14^{* *}$ \\
Alexitimia & & & & $.26^{* * *}$ \\
Vulnerabilidad Psicológica & & & & $.18^{* * *}$ \\
Estado Depresivo & & & & $.18^{* * *}$ \\
\hline
\end{tabular}

¿ 1 = Varón $2=$ Mujer $\quad{ }^{* * *} p<.001 \quad$ ** $p<.01{ }^{*} p<.05$

\section{DISCUSIÓN}

El presente estudio trató de identificar la forma como se relacionaba la alexitimia y sus distintas facetas con diversos procesos cognitivos y emocionales. En particular, nos interesó determinar la asociación de la alexitimia con la vulnerabilidad psicológica y la presencia de un estado anímico depresivo. Asimismo tratamos de establecer cuál era la contribución de la alexitimia a la hora de explicar la hostilidad, centrándonos en la expresión de la ira, el cinismo y la rumiación. En este sentido intentamos clarificar también si este conjunto 
Tabla 5. Análisis mediacional en la predicción del cinismo y la rumiaclón

\begin{tabular}{|c|c|c|c|c|}
\hline Cinismo & $\overline{\mathrm{R}^{2}}$ & $\bar{F}$ & $\overline{\text { gl }}$ & $\beta$ \\
\hline Género ${ }^{8}$ & .15 & $38,77^{* * *}$ & 2,427 & $-.08+$ \\
\hline Alexitimia & & & & $.38 * * *$ \\
\hline Género & .18 & $31,02^{* * *}$ & 3,427 & $-.14^{* *}$ \\
\hline Vulnerabilidad Psicológica & & & & $.32^{* * *}$ \\
\hline Estado Depresivo & & & & $.15^{* *}$ \\
\hline Género & .22 & $29,39 * * *$ & 4,427 & $-.12^{* *}$ \\
\hline Alexitimia & & & & $.23^{* * *}$ \\
\hline Vulnerabilidad Psicológica & & & & $.24 * * *$ \\
\hline Estado Depresivo & & & & $.09+$ \\
\hline \multicolumn{5}{|l|}{ Rumiación } \\
\hline Género? & .08 & $19,61^{* * *}$ & 2,427 & $-.12^{*}$ \\
\hline Alexitimia & & & & $.26 * * *$ \\
\hline Género &.$\overline{18}$ & $30,42^{* * *}$ & 3,427 & $-.17^{* * *}$ \\
\hline Vulnerabilidad Psicológica & & & & $.30^{* * *}$ \\
\hline Estado Depresivo & & & & $.16^{* *}$ \\
\hline Género & .18 & $23,32 * * *$ & 4,427 & $-.16^{* * *}$ \\
\hline Alexitimia & & & & $.07(\mathrm{~ns})$ \\
\hline Vulnerabilidad Psicológica & & & & $.27 * * *$ \\
\hline Estado Depresivo & & & & $.15^{* *}$ \\
\hline
\end{tabular}

$\Omega 1$ = Varón $2=$ Mujer $\quad$ *** $p<.001 \quad$ **p<.01 " $p<.05 \quad+=.06$

de relaciones podian estar afectadas, en alguna medida, por la vulnerabilidad psicológica y el estado anímico depresivo.

Los resultados hallados confirmaron nuestra primera hipótesis, al mostrar que la alexitimia se asociaba de forma positiva con la vulnerabilidad psicológica y el estado de ánimo depresivo. Además, de los tres componentes de la alexitimia, la dificultad para identificar los 
sentimientos fue el que se asoció en mayor grado con estas dos variables, siendo el pensamiento orientado al exterior el que menos relación tuvo.

Estos resultados resultan importantes en primer lugar porque, hasta el momento, ningún estudio ha abordado la conexión de la alexitimia con la vulnerabilidad psicológica. Además revelan que la alexitimia se relaciona con un estado de vulnerabilidad, caracterizado por el mantenimiento de creencias orientadas al perfeccionismo y a la necesidad de aprobación externa, y por un déficit en los recursos dirigidos a la adaptación (Sinclair y Wallston, 1999; Sinclair y Wallston, 2001). Este hecho pone de manifiesto, por tanto, que la persona alexitímica, además de tener dificultades en la autorregulación emocional, también podría contar con un deterioro en su capacidad para manejar el estrés.

De otra parte, los datos manifiestan que la alexitimia, y en particular la dificultad para identificar los sentimientos, pueden favorecer un estado afectivo disfórico, aun cuando el diagnóstico de la depresión no se halle consolidado. De esta manera es presumible que la persona alexitímica, al no ser capaz de diferenciar y modular su estado emocional negativo, mantenga un nivel permanente de anhedonia y de malestar emocional (Bagby et al., 1994; Lumley, 2000).

En cuanto a la relación y la capacidad de predicción de la alexitimia con respecto a la expresión del enfado, el cinismo y la rumiación, los resultados hallados apoyaron en gran medida nuestras hipótesis. En particular la alexitimia, considerada de forma global, predijo positivamente todos los criterios elegidos, siendo su contribución menor en el caso de la manifestación abierta de la ira.

El análisis de la contribución de la alexitimia a partir de sus componentes, proporcionó una visión más completa de su funcionamiento. Como esperábamos, y en la línea marcada por diferentes investigaciones (Ciarrochi et al., 2003; Quinton y Wagner, 2005; Waldstein et al., 2002), la dificultad relativa a la descripción de los sentimientos fue la faceta que se asoció en mayor grado con la ira suprimida, el cinismo y la rumiación. Sólo en el caso del enfado manifiesto esta dimensión tuvo un peso negativo, y menor que el mostrado por la dimensión referida a la dificultad para identificar los sentimientos. Estos datos podrían interpretarse considerando que, en la alexitimia, 
la falta de identificación de la experiencia emocional de la ira puede asociarse con una respuesta intensa y repentina de esta emoción. En contraste, el daño en la capacidad para elaborarla verbalmente puede conectarse con un manejo ineficaz de la misma, dirigiéndola contra uno mismo.

Asimismo merece la pena destacar la escasa capacidad predictiva del pensamiento dirigido al exterior, que sólo tuvo un peso débil en la predicción de la ira suprimida y el cinismo.

En consecuencia, observamos que dentro del espectro de la alexitimia no todas las facetas tenían el mismo peso, aspecto éste que amplía el conocimiento sobre la utilidad predictiva de la alexitimia con respecto a diferentes procesos cognitivos y emocionales.

A través de los datos obtenidos en este estudio, también tratamos de determinar si la relación de la alexitimia con los criterios estudiados estaba modulada, de algún modo, por la vulnerabilidad psicológica y el estado de ánimo depresivo.

Con respecto a la ira manifiesta, la contribución más importante fue la aportada por la vulnerabilidad, ya que la alexitimia no alcanzó el nivel de significación cuando se controló el efecto de la vulnerabilidad. A diferencia de lo reflejado en algunos estudios (Sandín et al., 1996; Waldstein et al., 2002), este dato sugiere que la alexitimia podría asociarse con la expresión abierta de la ira cuando se acompaña de un estado subjetivo de vulnerabilidad, y de falta de recursos propios para manejar las situaciones.

Por otro lado, la relación de la alexitimia con la rumiación se vio influida significativamente por la vulnerabilidad y el estado de ánimo depresivo. Este resultado podría explicarse teniendo en cuenta que la persona alexitímica no dispone de capacidad suficiente para diferenciar y simbolizar verbalmente las emociones relacionadas con la hostilidad. De esta forma el control de la expresión de dichas emociones, así como el control de la conducta agresiva mediante el pensamiento recurrente de la rumiación, tendrian lugar al estar presentes un estado anímico disfórico, junto con una serie de actitudes disfuncionales referidas a la autocrítica y a la falta de aceptación incondicional de uno mismo.

Finalmente nuestros resultados revelaron también que, una vez controlado el efecto de la vulnerabilidad y el estado de ánimo, la 
alexitimia seguía prediciendo la ira suprimida y el cinismo. Parece, por lo tanto, que la alexitimia se asocia con un conflicto en la regulación y expresión del enfado, tendiendo a su supresión y al desarrollo de una visión más hostil de los demás. La inhibición del enfado podría, además, verse mantenida por el mantenimiento de un estado depresivo crónico, y por una excesiva necesidad de aprobación externa. En apoyo a este argumento, algunos estudios han indicado que el individuo alexitímico cuenta con un nivel de apoyo social más bajo, y tiende a establecer unas relaciones interpersonales más débiles o distantes (Haviland y Reise, 1996; Lumley, Stettner y Wehmer, 1996).

El presente estudio cuenta con ciertas limitaciones que deben ser mencionadas. En primer lugar todas las medidas empleadas fueron autoinformadas, lo cual pudo haber aumentado la magnitud de las relaciones obtenidas. Por este motivo, habrán de incorporarse en futuros estudios otras medidas más objetivas, como índices conductuales o fisiológicos, e informes aportados por personas cercadas al participante, para precisar mejor la repercusión de la alexitimia sobre las variables criterio elegidas. Por otro lado la muestra seleccionada en nuestro estudio estuvo compuesta de personas jóvenes, ya que un $66 \%$ de los participantes tuvo una edad inferior a 40 años. Esta restricción sesga de alguna manera la posibilidad de generalizar los resultados a otras poblaciones con mayor rango de edad. Por último el planteamiento de un estudio transversal, dificulta el establecimiento de relaciones causales entre la alexitimia y los factores cognitivos y emocionales estudiados. El empleo de una metodología longitudinal contribuiría a discernir si la alexitimia puede derivarse también del mantenimiento de un conjunto de creencias que denoten vulnerabilidad psicológica, o de una actitud hostil y suspicaz hacia los demás.

En definitiva, a partir de la presente investigación, se puede destacar que la alexitimia se vincula con características asociadas a un bajo nivel de ajuste personal, y a un estado afectivo más negativo. Por otra parte, el conocimiento de las relaciones entre la alexitimia, la supresión de la ira y la hostilidad, abre nuevas vías para detectar las implicaciones de este constructo en el ámbito de la salud física y emocional. 


\section{REFERENCIAS BIBLIOGRÁFICAS}

Bagby, R.M., Parker, J.D.A., y Taylor, GJ. (1994). The twenty-item Toronto alexithymia scale-l. Item selection and cross-validation of the factor structure. Joumal of Psychosomatic Research, 41, $23-32$.

Bagby, R.M., Taylor, G.J., y Parker, J.D.A. (1997). The nomological domain of the alexithymia construct. En A. Vingerhoets, F. van Bussel y J. Boelhouwer (Eds.), The (non) expression of emotions, (pp.95102). Tilburg: Tilburg University Press.

Baron, R.M., y Kenny, D.A. (1986). The moderator-mediator variable distinction in social psychological research: Conceptual, strategic, and statistical considerations. Journal of Personality and Social Psychology, 42, 5-37.

Bermúdez, J. (1993). Adaptación española de la escala de DisipaciónRumiación: Datos preliminares. Informe del Departamento de Psicologia de la Personalidad. Madrid: UNED.

Bermúdez, J., Fernández, E., y Sánchez-Elvira, A. (1992). La Escala de Hostilidad (Ho) de Cook-Medley (Ho) para muestras españolas. Informe no publicado del Departamento de Psicología de la Personalidad. Madrid: UNED

Caprara, G.V. (1986). Indicators of agression: The DissipationRumination Scale. Personality and Individual Differences, 6, 763769

Ciarrochi, J., Scott, G, Deane, F.P., y Heaven, P.C.L. (2003). Relations between social and emotional competence and mental health: $A$ construct validation study. Personality and Individual Differences, 35, 1947-1963.

Cook , W.W., y Medley, D.M. (1954). Proposed hostility and pharisaicvirtue scales for the MMPI. Journal of Applied Psychology, 38, 414418.

Eizaguirre, A.E., Saenz de Cabezón, A.O., Alda, I.O., Olariaga, L.J., \& Juaniz, M. (2004). Alexitimia and its relationships with anxiety and depression in eating disorders. Personality and Individual Differences, 36, 321-331.

Fortune, D.G., Richards, H.L., Griffiths, C.E.M., y Main, C.J. (2002).

Psychological stress, distress and disability in patients with psoriasis: Consensus and variation in the contribution of illness perception, 
coping and alexithymia. British Journal of Clinical Psychology, 41, 157-174.

Handelsman, L., Stein, J.A., Bernstein, D.P., Oppenheim, S.E., Rosenblum, A., \& Magura, S. (2000). A latent variable analysis of coexisting emotional deficits substance abusers: Alexithymia, hostility and PTSD. Addictive Behaviors, 25, 423-428.

Haviland, M.G, y Reise, S.P. (1996). A California Q-set alexithymia prototype and its relationship to ego-control and ego-resiliency. Journal of Psychosomatic Research, 41, 597-608.

Helmers, K.F., y Mente, A. (1999). Alexithymia and health behaviors in health male volunteers. Journal of Psychosomatic Medicine, 47, 635-645.

Houston, B.K., y Vavak, C.R. (1991). Cynical hostility: Developmental factors, psychosocial correlates, and health behaviors. Health Psychology, 10, 9-17.

Knight, R.G, Chisholm, B.J., Paulin, J.B., y Waal-Manning, J. (1988). The Spielberger Anger Expression Scale: Some psychometric data. British Journal of Clinical Psychology, 27, 279-281.

Krystal, H. (1982). Alexithymia and the effectiveness of psychoanalytic treatment. International Joumal of Psychoanalitic Psychotherapy, 9, 353-378.

Lesser, I.M. (1981). A review of the Alexithymia concept. Psychosomatic Medicine, 43, 531-541.

Linden, W., Lenz, J.W., y Sotssel, C. (1996). Alexitimia, defensiveness and cardiovascular reactivity to stress. Joumal of Psychosomatic Research, 41, 575-583.

Luminet, O., Bagby, R.M., y Taylor, GJ. (2001). An evaluation of the absolute and relative stability of alexithymia in patients with major depression. Psychotherapy and Psychosomatics, 70, 254-260.

Lumley, M.A. (2000). Alexithymia and negative emotional conditions. Journal of Psychosomatic Research, 49, 51-54.

Lumley, M.A., Stettner, L., y Wehmer, F.(1996). Alexithymia, social support and health problems. Journal of Psychosomatic Research, 41, 519-530.

Marchesi, C., Brusamonti, E., y Maggini, C. (2000). Are alexithymia, depression, and anxiety distinct constructs in affective disorders? Journal of Psychosomatic Research, 49, 43-49. 
Martin, C.R., y Thompson, D.R. (2000). A psychometric evaluation of the hospital anxiety and depression scale in coronary care patients following acute myocardial infarction. Psychology, Health and Medicine, 5, 193-201.

Modestin, J., Furrer, R., y Malti, T. (2004). Study on alexithymia in adult non-patients. Journal of Psychosomatic Research, 56, 707-709.

Pelechano, V. (2000). Cuestionario de hostilidad y malevolencia (HOSTYMAL). Análisis y Modificación de Conducta, 26, 780-815.

Pérez-García, A., Rueda, B., Sanjuán, P., y Ruiz, M.A.. Spanish adaptation of the psychological vulnerability scale: Normative data and convergent validity in health context. Póster presentado en la $13^{\text {th }}$ European Conference of Personality. Grecia, 006.

Posse, M., Backenroth-Ohsano, G, Häkanson, C.E., y Hällström, T. (2001). Alexithymia and psychiatric symptoms in a population of nursery workers: A study using the 20-item Toronto Alexithymia scale. Social Behavior and Personality, 29, 491-502.

Quintana, J.M., Padierna, A., Esteban, C., Arostegui, I., Bilbao, A., y Ruiz, I. (2003). Evaluation of the psychometric characteristics of the Spanish version of the Hospital Anxiety and Depression scale. Acta Psychiatrica Scandinavica, 107, 216-221.

Quinton, S., y Wagner, H.L. (2005). Alexithymia, ambivalence over emotional expression and eating attitudes. Personality and Individual Differences, 38, 1163-1173.

Rief, W., Heuser, J., y Fichter, M.M. (1996). What does the Toronto Alexithymia scale TAS-R measure?. Journal of Clinical Psychology, $52,423-429$.

Rueda, B. (2003). Competencia percibida y salud: Aproximación general y aplicación a la enfermedad cardiovascular. Tesis doctoral no publicada. Madrid: UNED

Saarijärvi, S., Salminen, J.K., y Toikka, T.B. (2001). Alexithymia and depression: A 1-year follow-up study in outpatients with major depression. Journal of Psychosomatic Research, 51, 729-733.

Sandín, B., Chorot, P., Santed, M.A., y Jiménez, P. (1996). Dimensiones de alexitimia y estados emocionales de ansiedad, depresión e ira. Psiquis, 17, 37-45.

Sifneos, P.E. (1972). Psychotherapy and emotional crisis. Cambridge: Hardvard University Press. 
Sinclair, V.G., y Wallton, K.A. (1999). The development and validation of the psychological vulnerability scale. Cognitive Therapy and Research, 23, 119-129.

Sinclair, V.G., y Wallston, K.A. (2001). Predictors of improvement in a cognitive-behavioral intervention for women with rheumatoid arthritis. Annals of Behavioral Medicine, 23, 291-297.

Smith, T.W., Glazer, K., Ruiz, J.M. y Gallo, L.C. (2004). Hostility, Anger, Aggressiveness, and Coronary Heart Disease: An Interpersonal Perspective on Personality, Emotion, and Health. Journal of Personality, Vol. 72 Issue 6, 1217-1271.

Spielberger, C.D., Johnson, E.H., Russell, S.F., Crane, R.J., Jacobs, G.A., y Worden, T.J. (1984). The experience and expression of anger: Construction and validation of an anger expression scale: En M.A. Chesney, S.E. Goldston, y R.H. Rosenman (Eds.), Anger and hostility in behavioural medicine (pp.5-30). New York: Hemisphere / McGraw.

Taylor, G.J., Bagby, R.M., y Luminet, O. (2000). Assessment of alexithymia: Self-report and observer-rated measures. En J.D.A. Parker and R. Bar-On (Eds.), The handbook of emotional intelligence (pp. 301-319). San Francisco, CA: Jossey Bass.

Taylor, G.J., Bagby, R.M., y Parker, J.D.A. (1997). Disorders of Affect Regulation: Alexithymia in medical and psychiatric illness. Cambridge: Cambridge University Press.

Taylor, G.J., Ryan, D.P., y Bagby, R.M. (1985). Toward the development of a new self-report Alexithymia scale. Psychotherapy and Psychosomatics, 44, 191-199.

Yücel, B., Kora, K., Özyalcín, S., Alcalar, N., Özdemir, O., y Yücel, A. (2001). Depression, automatic thoughts, alexithymia, and assertiveness in patients with tension-type headache. Headache, 42, 194-199.

Waldstein, S.R., Kauhanen, J., Neumann, S.A., y Katzel, L.I. (2002). Alexithymia and cardiovascular risk in older adults: psychosocial, psychophysiological, and biomedical correlates. Psychology and Health, 17, 597-610.

Zigmond, A.S., y Snaith, R.P. (1983). The Hospital Anxiety and Depression Scale. Acta Psychiatrica Scandinavica, 67, 361-370. 